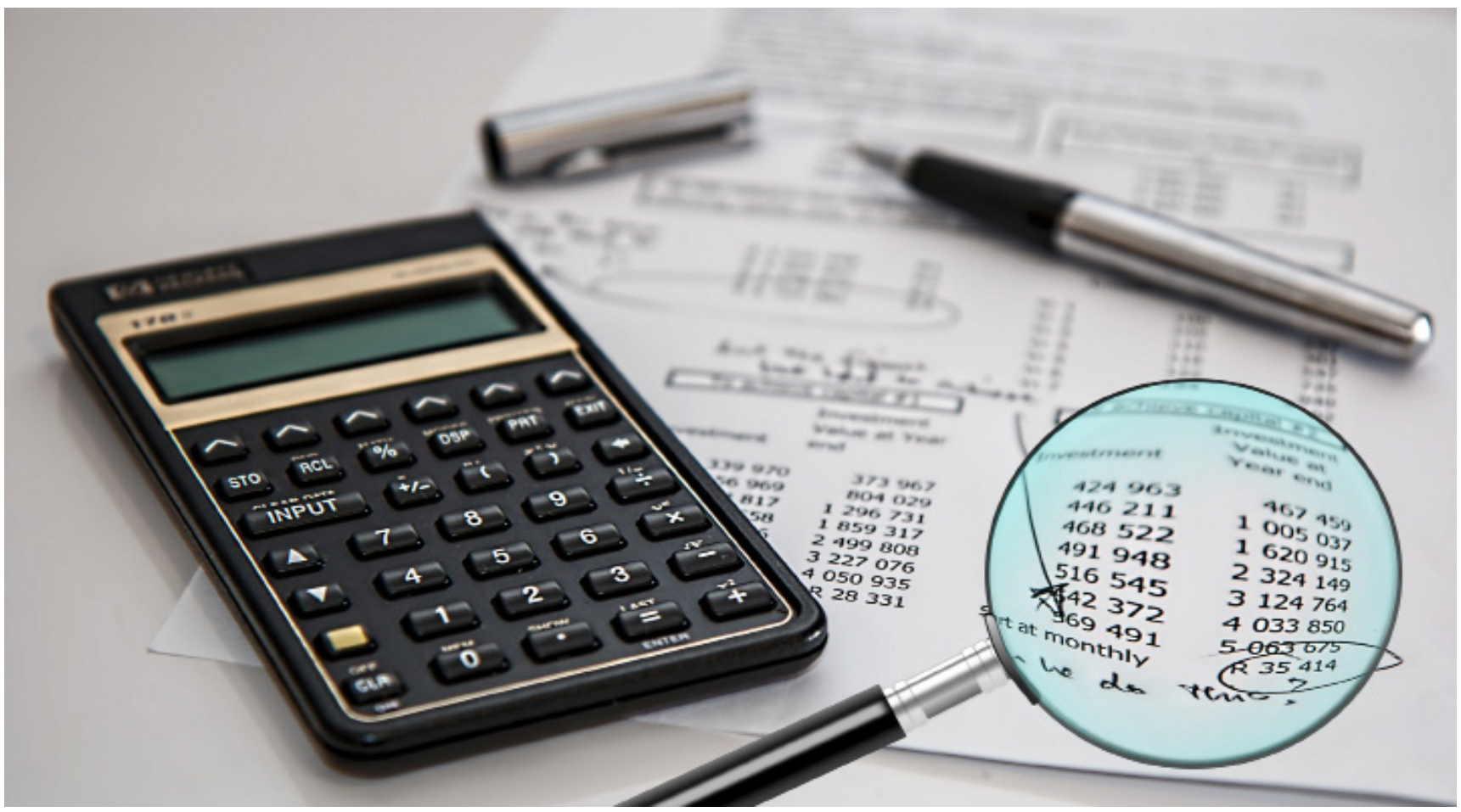

\title{
El control social a lo público educa, mejora nuestra calidad de vida y es responsabilidad de todos.
}

\section{Social control of the public educates, improves our quality of life and is the responsibility of everyone.}

\section{Uriel Quintero-Lobo}

Especialista en Gestion Pública, gp_uquintero@fesc.edu.co,

https://orcid.org/0000-0002-5585-4121, Fundación de Estudios Superiores

Comfanorte, Cucuta, Colombia.

Diana Paola Beltrán- Quintero

Especialista en Gestion Pública, gp_dp_beltran@fesc.edu.co

https://orcid.org/0000-0001-8272-6830,

Fundación de Estudios Superiores Comfanorte, Cucuta, Colombia.

María Alejandra Afanador

Especialista en Gestion Pública, ma.afanador@fesc.edu.co

https://orcid.org/0000-0001-7106-5301,

Fundación de Estudios Superiores Comfanorte, Cucuta, Colombia. 
Resumen

El presente trabajo se podrá observar un estudio estadístico con su respectivo análisis sobre la situación real y cierta que está sucediendo en la ciudad de San José de Cúcuta, en lo concerniente a la baja Participación Ciudadana y el Control Social a lo Público, por parte de los Jóvenes estudiantes del nivel media, estudio y proyecto a través del cual se logró evidenciar que la mayoría de jóvenes estudiantes no tienen conocimiento de las normatividad existente relacionada con la participación ciudadana y el control social a lo público. Circunstancia que les impide abordar temas como el impacto de las obras que hoy se llevan a cabo en la ciudad, de igual sentido poder abarcar temas de importantes como el desempleo, la violencia, la inseguridad y la falta de obras en sus comunidades, situaciones que las encuestas aplicadas a los estudiantes del colegio San Francisco de Sales, han dejado en claro y como un fuente para alertar a los diferentes órganos de control del Estado como lo son la Contraloría, la Personería, la Defensoría del Pueblo, la Alcaldía de Cúcuta; a través de sus secretarias de desarrollo social y la secretaria de educación, deben aplicar las recomendaciones y planteamientos que se presentan en este documento como una herramienta valedera, para forjar mejores jóvenes en sus entorno social y con conocimientos en participación ciudadana y control social.

Palabras clave: Veeduría, control social, políticas públicas, seguimiento, monitoreo, participación ciudadana.

\section{Abstract}

The present work will be able to observe a statistical study with its respective analysis on the real and certain situation that is happening in the city of San José de Cúcuta, concerning the low Citizen Participation and the Social Control to the Public, on the part of the Young students of the average level, study and project through which it was managed to demonstrate that most of young students do not have knowledge of the existing regulations related to the citizen participation and the social control to the public. This prevents them from addressing issues such as the impact of the works being carried out in the city today, as well as being able to address important issues such as unemployment, violence, insecurity and the lack of works in their communities, situations that the surveys applied to students at the Colegio San Francisco de Sales have made clear and as a source to alert the various state control bodies such as the Contraloría, the Personería, the Defensoría del Pueblo, the Alcaldía de Cúcuta; Through their Secretaría de Desarrollo Social and the Secretaría de Educación, they should apply the recommendations and proposals presented in this document as a valid tool to forge better young people in their social environment and with knowledge in citizen participation and social control.

Keywords: Oversight, social control, public policies, follow-up, monitoring, citizen participation. 
Introducción

En la actualidad los problemas originados por la corrupción en el mundo son los responsables de grandes males que afectan a la sociedad y que absorbe temas como el desempleo, la violencia, la inseguridad, el enriquecimiento ilícito de administradores y gobernantes, daños al medio ambiente y muchos otros que repercuten directamente en la calidad de vida del ciudadano. Denótese que parte importante de la solución está en una mayor y más activa participación ciudadana en la toma de decisiones, así como en la ejecución de los planes y programas que se ejecutan en la administración pública. Este monitoreo es vital para la supervisión efectiva, y la obtención de resultados óptimos, en la calidad de vida de la localidad [1-3].

Según [4] la Ley Estatutaria de Ciudadanía Juvenil "dio mayor empoderamiento al joven dentro de sus espacios de participación y representación, convirtiendo los consejos de juventud en escenarios más que simplemente consultivos para convertirlos en instancias de decisión de carácter vinculante respecto de sus decisiones"; sin embargo, existe una especie de apatía a este respecto, los jóvenes no creen en el sistema y avalados por la crisis de salud actual que vive el mundo se dedican a sus propios intereses olvidando este, que a su vez se reflejaría en el mejoramiento de su calidad de vida y en la de sus comunidades [5].

Esta investigación sirve como una herramienta valedera, para despertar en los jóvenes, mayor atención a su entorno social y mejorar sus conocimientos en participación ciudadana y control social a lo público. Elementos básicos para poder hablar de sentido de pertenencia y mejoramiento de calidad de vida de la juventud que redundará en el beneficio del colectivo y social del entorno al que se quiere mejorar.

\section{Marco teórico}

El control Social a lo Público y la Participación Ciudadana, debe serun matrimonio que no debe fallar, por lo que es necesario entender que la forma en que las sociedades se han organizado entre administrados y administradores, es decir entre quienes gobiernan y quienes son gobernados, ha variado históricamente y se pueden identificar varias formas de gobierno en diferentes momentos de la humanidad [69].

Colombia se puede afirmar que es un Estado social de derecho, organizado en forma de República unitaria, descentralizada, con autonomía de sus entidades territoriales, democrática, participativa y pluralista, fundada en el respeto de la dignidad humana, en el trabajo y la solidaridad de las personas que la integran y en la prevalencia del interés general. Sin embargo, la sociedad colombiana en la actualidad pasa por uno de sus momentos más críticos en términos de participación política [10]. Según cifras de la Registradora Nacional del Estado Civil, Durante la primera vuelta de la elección presidencial de 2014, el $59.3 \%$ de los colombianos que podían hacerlo no votaron. Igualmente, en el plebiscito de 2016 para saber si los colombianos avalan o no el Acuerdo de Paz entre el Gobierno y las Farc, la cifra de abstención fue del 62,57 \% [11].

Es importante resaltar porque las Instituciones se encuentran en crisis, la falta de credibilidaden la institucionalidad por parte de la ciudadanía “(...) es decir, en las que menos confianza les genera a los colombianos, la Justicia, las altas cortes y elCongreso de la República encabezan esa lista. De acuerdo con la encuesta de Ipsos, el $77 \%$ cree que la Justicia no está haciendo las cosas correctamente para que el país salga adelante [12]. El $75 \%$ cree que el trabajo que se está haciendo en el Senado y en la Cámara de Representantes no es confiable." La falta de credibilidad casos de corrupción en el sector 


\section{4}

público y el sector privado aumentos de la percepción de prácticas indebidas respecto a los pagos para agilizar trámites en el sector público (65\%) y con respecto a contribuciones privadas a campañas y partidos políticos (54\%)" [13]. Esto ha generado desinterés y la indiferencia por parte de la ciudadanía en la esfera de lo público. Por tal razón, no es un mal menor que la baja participación ciudadana posibilite el cuestionarse ¿Qué tan legítima termina siendo una democracia con índices tan bajos de participación? ¿Es posible mejorar la participación ciudadana más allá de su forma electoral, es decir de la democracia representativa para pasar a una democracia más participativa? ¿Es posible incidir en nuevas generaciones de ciudadanos (Jóvenes) para que asuman un papel más activo, permitiendo el fortalecimiento la democracia? ¿Qué metodología pedagógica es más acorde para dinamizar en la juventud colombiana para su formación en democracia participativa? ¿Qué metodología de formulación de un proyecto social es más acertada si se pretende realizar un proyecto que contribuya en la capacitación de competencias ciudadanas como la participación?

\section{Materiales y métodos}

El tipo de investigación que se aplicó fue la descriptiva, con una metodología mixta con enfoque dominante cualitativo con lo que se buscó dar respuesta a la investigación. El modelo cualitativo se soportó a través de la realización de 5 entrevistas llevadas a cabo a las personas involucradas directamente con los organismos de control y el análisis de los informes de gestión publicados en los últimos dos periodos de gobierno; lo cual permitió conocer la percepción actual frente al bajo nivel de participación ciudadana y el fenómeno de corrupción más repetitivo.

En este sentido, se consideró relevante para la presente investigación el desarrollo de la metodología cualitativa, para conocer los diferentes mecanismos y/o estrategias que se emplean para la Promoción y Participación Ciudadana en el contexto interno y externo que acompaña a la Institución Educativa (I.E) San Francisco de Sales.

Para el desarrollo de la investigación cualitativa, se empleó la entrevista semiestructurada, descrita como fuente primaria que permitió recolectar información de los funcionarios relacionados directamente con los organismos de control. Para el desarrollo del modelo de investigación cuantitativa se aplicó una encuestaalosEstudiantesdelal.E.SanFrancisco de Sales, instrumento que permitió identificar el estado actual del conocimiento sobre los diferentes mecanismos de Participación Ciudadana, como son las veeduría y control social a lo público, lo cual permitió conocer las estrategias de Participación Ciudadana; de igual forma, se obtuvo la percepción que tienen los estudiantes sobre los fenómenos de corrupción que afectan la ciudad de Cúcuta.

Cabe resaltar, que de igual manera se empleó como instrumento para la medición cuantitativa gráficas: de barras y de línea de tendencia.

Como referentes para la recolección de la información se tuvieron en cuenta las siguientes fuentes:

a. Observatorio de Transparencia y nticorrupción 2008 y 2018.

b. Sistema Penal Oral Acusatorio - SPOA Fiscalía General de la Nación 2008 - 2018.

c. Sistema Integral de Auditoria - SIA Auditoria General de la Republica.

d. Sistema de Información de Registro de Sanciones y Causas de Inhabilidad - SIRI Procuraduría General De La Nación 2016 -2018 
e. Alcaldía de Cúcuta Informes de Gestión 2016-2019

\section{f. Cámara de Comercio de Cúcuta}

g. Informes de la Contraloría Municipal de Cúcuta.

De ésta manera se detectaron las necesidades reales y actuales de los estudiantes, así como la propuesta de gestión pública que respondió al mejoramiento del indicador de Participación Ciudadana pertinencia, eficacia, eficiencia y buen gobierno de la I.E. San Francisco de Sales.

\section{Población y muestra}

El modelo de investigación diseñado se aplicó al universo objeto de estudio, que correspondió a la Institución Educativa San Francisco de Sales del Municipio San José de Cúcuta y Organismos de Control, que para la Investigación correspondió a 184 Estudiantes y 23 funcionarios públicos a quienes se aplicó, encuestas y entrevistas referidos al desempeño de sus funciones, en lo relacionado con Organismos de Control del Estado que laboran en el Municipio de Cúcuta.

Cabe resaltar que la muestra para la aplicación de la entrevista fue de tipo no probabilístico. Al interactuar con los actores mencionados anteriormente, y verificar los indicadores obtenidos fue posible identificar los diferentes delitos de corrupción más cometidos, así como los delitos contra los mecanismos de Participación Democrática, las sanciones fiscales, las sanciones disciplinarias y las estrategias implementadas por el gobierno local en materia de Promoción y Participación Ciudadana, procesos de veeduría y control social a los publico, igualmente el número de veedurías creadas.
Resultados

Esta investigación permitió identificar el estado actual sobre el conocimiento de los diferentes mecanismos de Participación Ciudadana en los diferentes procesos de veeduría y control social a lo público, conocer sus estrategias de Participación Ciudadana; de igual forma, conocer la percepción que tienen sobre los fenómenos de corrupción que afectan la ciudad. En este contexto, en el artículo se presentan los resultados referidos a la participación ciudadana, el desinterés y la apatía a realizar control social a lo público y los fenómenos, como la corrupción, que afectan la institucionalidad pública. Para ello se recolectó de manera narrativa argumentativa los hallazgos y resultados de la investigación a través de la ejecución de cuatro objetivos específicos:

1. Causas que generan el bajo nivel de participación ciudadana en los procesos de veeduría y control social a la gestión pública.

2. Análisis de la situación actual relacionada con el bajo nivel de participación ciudadana y el fenómeno de corrupción, a partir de indicadores e informes de gestión publicados por los organismos de control en los últimos dos períodos de gobierno nacional.

3. Acciones estratégicas implementadas por el gobierno local en los últimos dos períodos, en materia de promoción y participación ciudadana en los procesos de veeduría y control social a lo público.

4. Propuesta de mecanismos y/o alternativas para mejorar el indicador de participación ciudadana y control social a lo público, a partir de los adolescentes y jóvenes de educación media, a través de la Implementación del Manual del Contralor Estudiantil, y material didáctico como dispositivas en las cuales se capacita y seda conocer los argumentos 


\section{6}

constitucionales y legales sobre participación ciudadana, control social a lo público, como funciona la figura del contralor estudiantil, para que sirven la veedurías ciudadanas $y$ el funcionamiento del aparato estatal en Colombia a través de sus órganos de control del Estado.

A través de esta investigación se logró evidenciar que la mayoría de jóvenes estudiantes no tienen conocimiento de la normatividad existente relacionada con la participación ciudadana y el control social a lo público. Circunstancia que les impide abordar temas como el impacto de las obras que hoy se llevan a cabo en la ciudad, y la forma de gobernabilidad que ha venido desarrollándose en la ciudad, situaciones resultantes del impacto en la baja participación ciudadana, y trasciende a temas de política pública de competencia de la institucionalidad, que a los gobernantes locales y de turno poco afectan en sus planes de gobierno.

En sentido contario se observaron los resultados de las opiniones de los educandos que se refieren al desempleo, la violencia, la inseguridad y la falta de obras en sus comunidades, como necesidad de articulación y actuar armónicamente entre Gobierno y Gobernados, opiniones que en esta investigación, los estudiantes del colegio San Francisco de Sales, han dejado en claro y como una fuente importante para alertar a los diferentes órganos de control del Estado como lo son la Procuraduría, la Contraloría, la Personería, la Defensoría del Pueblo, y especialmente la Administración Municipal Alcaldía de Cúcuta, yla Secretaria de Educación Municipal, para que actúen en beneficio del control social participativo.

Con el objeto de identificar las causas que generan el bajo nivel de participación ciudadana en los procesos de veeduría y control a la gestión pública, es oportuno y pertinente tener en cuenta estos interrogantes. ¿Qué incidencia tiene la falta de cultura ciudadana, el sentido de pertenencia y el control social participativo de la comunidad en las Entidades Públicas e Instituciones Educativas en la Ciudad de San José de Cúcuta?

Este análisis requirió llevar a cabo, la creación de instrumentos de medición, que permitieran identificar las causas que generan el bajo nivel de participación ciudadana en los procesos de veeduría y control a la gestión pública, ya que no se logró obtener un verdadero estudio, estadísticas e indicadores por parte de la AdministraciónMunicipalatravés delaSecretaria de Desarrollo Social y otros organismos como Personería Municipal, Contraloría Municipal. Puesto que la información existente se limita al cumplimiento de metas e indicadores de sus planes estratégicos e institucionales a cuatro años y el respectivo plan de acción anual, en concordancia con los objetivos institucionales, las metas estratégicas y los compromisos adquiridos previamente, como lo establece la normatividad existente que regula la materia [14-16].

Frente a la pregunta ¿Cuáles son los principales problemas que afectan nuestra ciudad?, se logró evidenciar que de los 184 Estudiantes de la I.E. San Francisco de Sales, encuestados el $84,8 \%$ de los estudiantes considera el Desempleo como el principal problema que afecta la ciudad de San José de Cúcuta, lo cual es visible ante toda la comunidad Cucuteña, porque no se logró obtener una estrategia del gobierno municipal, que pudiese mitigar esta problemática, con el auspicio de situaciones geográfica, social y políticas que le aquejan por ser una ciudad fronteriza, y otros fenómenos como el poco desarrollo industrial y empresarial a causa de la falta de inversión y proyectos, fenómenos que desarrollan el desempleo. Como segundo problema los estudiantes, creen que afecta la ciudad con un porcentaje del $81 \%$ es el 
de corrupción debido a que es visible el mal manejo de los recursos públicos por parte de los administradores públicos, además del poco compromiso con el desarrollo progresivo de la ciudad; y como tercer problema que afecta la ciudad es con el $58,7 \%$ es la violencia debido a los actos delincuenciales que se presentan día a día y la inseguridad existente en la ciudad, junto al corredor fronterizo y la constante migración, hace ver más vulnerable la ciudad en materia de violencia. En relación con la pregunta ¿Qué mecanismos de Promoción y participación ciudadana aplica?. La I.E. San Francisco de Sales, aplica como mecanismo de promoción y participación ciudadana, según el 59,2\% de los encuestados, charlas de orientación e información sobre el tema, seguidamente con el 41,8\% a rendición de cuentas, el $35,9 \%$ capacitaciones, y por último el $26,6 \%$ respondió que realiza conferencias.

Otra pregunta importante que se planteo fue, ¿cuál es su percepción sobre la incidencia de actos de corrupción en las entidades públicas?, donde el $38 \%$ de los estudiantes respondieron que "afecta a la ciudad en el factor socio-cultural y económico", según el porcentaje del 16.3 \% consideró que otro acto que favorece la corrupción corresponde a, "la carencia de valores, principios y educación de los gobernantes", al igual que: "Se debe a la mala elección de estos", el 15,2\% manifestó que "la corrupción incide en el mal manejo de los recursos" y por último el $14,1 \%$ consideran que "la corrupción siempre ha existido y no han podido disminuirla". Respecto a la pregunta ¿cuáles considera como fenómenos de corrupción en la ciudad de Cúcuta?, el 43,5\% de los estudiantes consideran como principal fenómeno de corrupción "la malversación de los recursos públicos", como segundo fenómeno con un 37\% "contratación estatal con intereses particulares", el 11,4\% considera "el soborno, extorsiones, fraudes" y por último el 8,2\% manifestó que "el sobre costo de los servicios públicos" son fenómenos de corrupción.

En la tabla 1 se observa la sistematización a la pregunta ¿Cuáles considera que son los principales problemas que afectan nuestra ciudad?.

Tabla 1 entrevista funcionarios relacionados con los órganos de control

\begin{tabular}{|c|c|c|}
\hline Entrevistados & Respuesta & \multirow{6}{*}{ 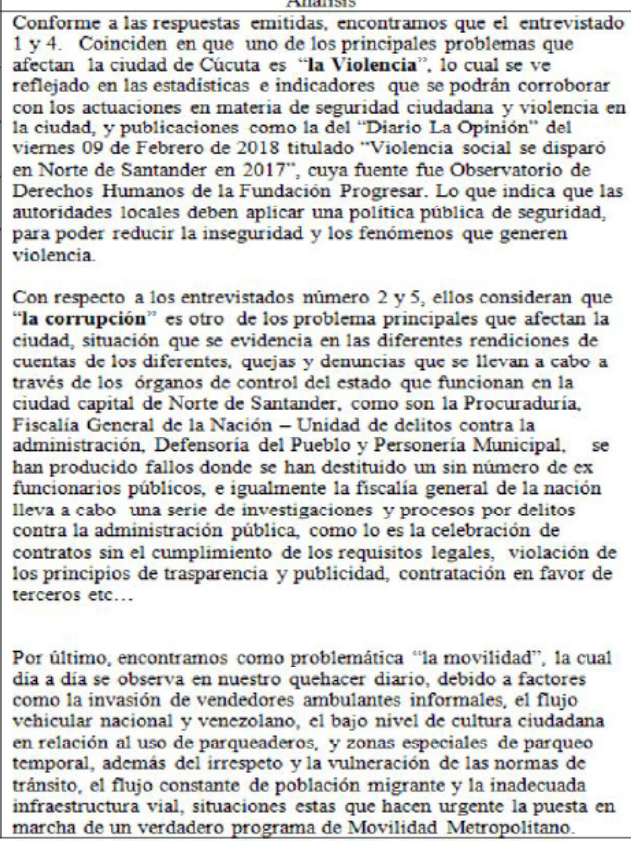 } \\
\hline Entrevistado 1 & Violencia & \\
\hline Entrevistado 2 & Corrupción & \\
\hline Entrevistado 3 & Movilidad & \\
\hline Entrevistado 4 & Violencia & \\
\hline Entrevistado 5 & Corrupción & \\
\hline
\end{tabular}


El control social a lo publico educa, mejora nuestra calidad de vida, y es responsabilidad de todos.

\section{8}

Respecto a la pregunta ¿cuál es su percepción sobre la incidencia de actos de corrupción en las entidades públicas?, (tabla 2):

Tabla 2 entrevista percepcion incidencias de los actos de la corrupcion

\begin{tabular}{|c|c|c|}
\hline Entrevistados & Respuesta & Análisis \\
\hline Entrevistado 1 & $\begin{array}{l}\text { Mal manejo del } \\
\text { presupuesto }\end{array}$ & \multirow{5}{*}{$\begin{array}{l}\text { De las Respuestas emitidas por los funcionarios de los } \\
\text { órganos de control, encontramos que estas se derivan } \\
\text { del fenómeno de corrupción a nivel Pais, como } \\
\text { claramente se puede apreciar de los indicadores y } \\
\text { fuentes consultadas en la investigación realizada como } \\
\text { fueron: Observatorio de Transparencia y } \\
\text { Anticorrupción 2008 y 2018, Sistema Penal Oral } \\
\text { Acusatorio - SPOA Fiscalia General de la Nación } \\
2008 \text { - 2018, Sistema Integral de Auditoria - SIA } \\
\text { Auditoria General de la Republica, Sistema de } \\
\text { Información de Registro de Sanciones y Causas de de } \\
\text { Inhabilidad - SIRI Procuraduria General De La Nación } \\
2016 \text {-2018, Cúcuta, Informes de la Contraloria } \\
\text { Municipal de Cúcuta, e informes de gestión de la } \\
\text { Comisión Regional de Moralización de Norte de } \\
\text { Santander. }\end{array}$} \\
\hline Entrevistado 2 & $\begin{array}{l}\text { Falta de } \\
\text { conciencia por } \\
\text { parte de los } \\
\text { gestores } \\
\text { públicos }\end{array}$ & \\
\hline Entrevistado 3 & Falta de Ética & \\
\hline Entrevistado 4 & $\begin{array}{l}\text { Falta de } \\
\text { Educación }\end{array}$ & \\
\hline Entrevistado 5 & $\begin{array}{l}\text { Falta de } \\
\text { mecanismos de } \\
\text { control. }\end{array}$ & \\
\hline
\end{tabular}

En relación a la pregunta ¿cuáles serían sus aportes para construir mejores ciudadanos en nuestra colectividad? (tabla 3 )

Tabla 3 Entrevista aportes para construir mejores cuidadanos

\begin{tabular}{|c|c|c|}
\hline Entrevistados & Respuesta & \multirow{6}{*}{$\begin{array}{l}\text { Análisis } \\
\text { Respecto las respuestas emitidas por los entrevistados } 1 \text {, } \\
2,4 \text { y } 5 \text {, se obtiene que la palabra educación, marca el } \\
\text { derrotero y pauta a seguir como el instrumento idóneo, } \\
\text { eficaz y efectivo, que generara mejoramiento y calidad de } \\
\text { vida de todos los Jóvenes Educandos de la I.E. San } \\
\text { Francisco de Sales del Municipio de Cucuta y por ende el } \\
\text { mejoramiento continua del tejido social de este } \\
\text { municipio; que conllevará al logro y cumplimiento de las } \\
\text { necesidades básicas insatisfechas de una población que } \\
\text { requiere los esfuerzo de la institucionalidad publica y el } \\
\text { trabajo armonioso y coordinado entre gobernantes y } \\
\text { gobernados a través de la Cultura ciudadana, sentido de } \\
\text { pertenencia y el control social a lo público. }\end{array}$} \\
\hline Entrevistado 1 & $\begin{array}{l}\text { Fomentar la } \\
\text { educación a gran } \\
\text { escala }\end{array}$ & \\
\hline Entrevistado 2 & Mejor Educación & \\
\hline Entrevistado 3 & $\begin{array}{l}\text { Proyectos con } \\
\text { innovación social un } \\
\text { cambio que se necesita }\end{array}$ & \\
\hline Entrevistado 4 & $\begin{array}{l}\text { Más oportunidades en } \\
\text { empleo, educación, } \\
\text { vivienda. }\end{array}$ & \\
\hline Entrevistado 5 & $\begin{array}{l}\text { La educación es la } \\
\text { base de toda sociedad }\end{array}$ & \\
\hline
\end{tabular}

Ahora bien, conforme a los pronunciamientos reseñados y abordados se genera el planteamiento del problema, como lo es el fenómeno de la corrupción, pero a la vez plantean ideas valiosas para combatirla. Es necesario y posible que se deba ir más allá y empoderar la presencia del ciudadano para, de esta manera a través del monitoreo se conduzca a la transparencia y la rendición real de cuentas en todos los ámbitos de la gestión pública. Para ello, es necesario y oportuno el fortalecimiento también la responsabilidad subjetiva de cada uno de los funcionarios electos respecto de sus electores, e igualmente conceder participación efectiva a los ciudadanos en la toma de decisiones. O sea, procurar que el gobierno en todos los niveles y ámbitos sea verdaderamente responsable ante todos los ciudadanos. 
Esto debe avanzar hacia una democracia participativa que complemente la actual democracia representativa, y así poder empoderar a los jóvenes estudiantes hacia una cultura ciudadana de respeto y responsabilidad de todos por el debido cuidado a los recursos y bienes públicos.
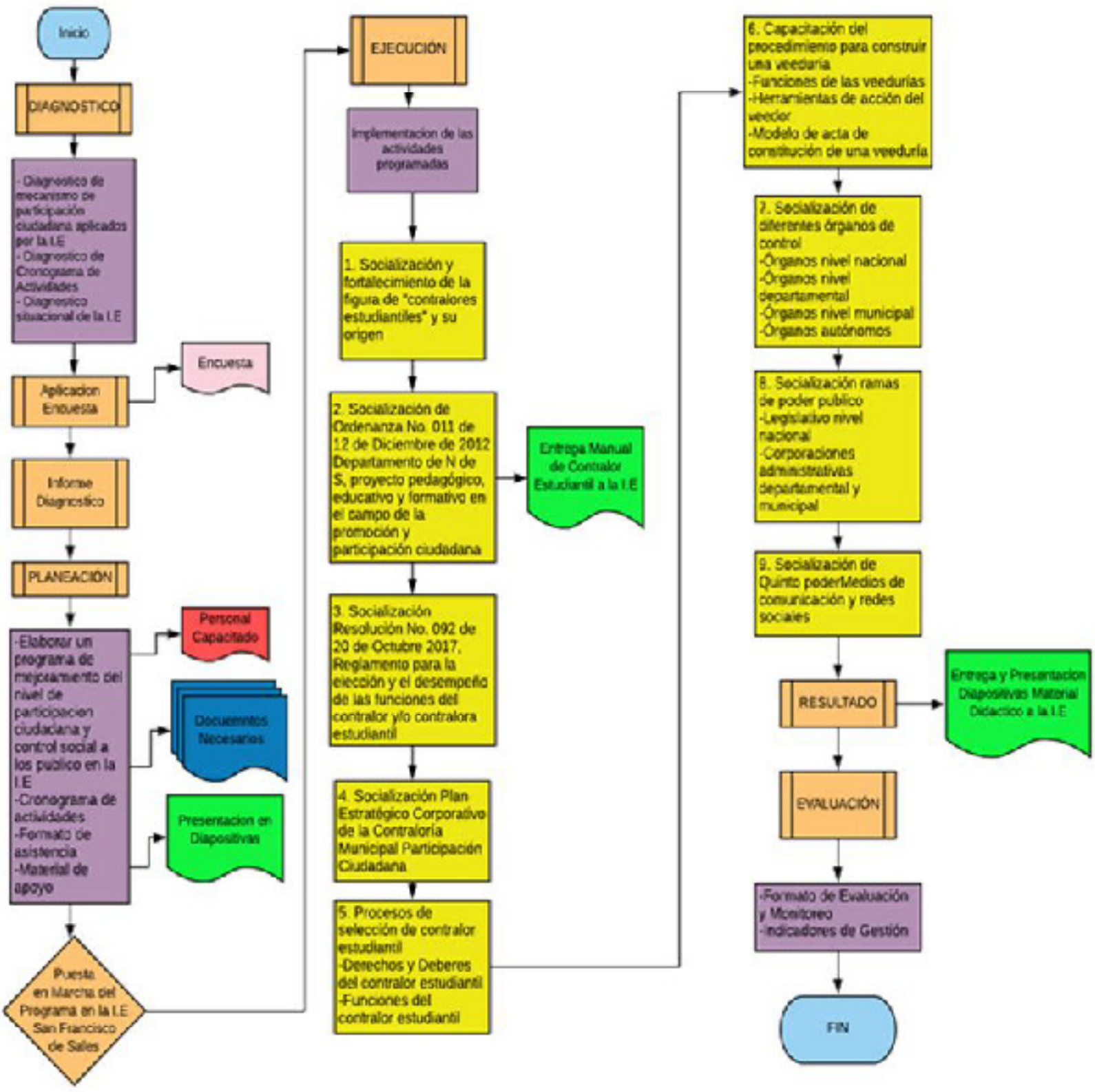

Figura 1. Flujograma de gestión pública - procesos para el mejoramiento de la participación ciudadana en las Instituciones Educativas. 


\section{0}

\section{Conclusiones}

Este estudio además identifica y demuestra verdaderamente, cuáles son las causas que generan el bajo nivel de participación ciudadana en los procesos de veeduría y control a la gestión pública, logrando evidenciar que el desinterés y la apatía hacia el sector público especialmente en la ciudad de San José de Cúcuta, confluyen como una falta de sentido de pertenencia de la colectividad, donde claramente se evidencia, que la mayoría de estudiantes tienen una conceptualización de ideas muy similares y perciben que los fenómenos de corrupción que afectan a la ciudad, los cuales vienen perjudicando ostensiblemente la región, puesto que el fenómeno de la corrupción en el sector público, se percibe como común e innato.

Llama la atención, que los estudiantes han manifestado "...que no se puede hacer nada", "que eso siempre ha existido". Por otra parte creen que hace falta mucho más control con el manejo de los recursos públicos, y hacen precisión al manifestar "que toda la sociedad lo sabe y lo permite", pero que esta situación es origen de la mala elección de los gobernantes porque carecen de valores, principios y educación, compendio de opiniones que merecen tener toda la atención tanto de la institucialidad publica, como de la academia y de sectores que pueden contribuir con la contraloría social como las ONG entre las cuales se puede destacar la organización Trasparencia por Colombia [16].

Otro de los fenómenos que estudia este trabajo, es la situación actual relacionada con el bajo nivel de participación ciudadana y el fenómeno de corrupción, a partir de indicadores e informes de gestión publicados por los organismos de control en los últimos dos períodos de gobierno nacional, sobre este importante aspecto de impacto se logró como resultadosatravés delasfuentes delossistemas de información de los diferentes órganos de control de estado, e indicadores que demuestran cómo avanzan las investigaciones penales, disciplinarias y procesos de responsabilidad fiscal frente a hechos 0 actuaciones, originadas por el fenómeno de la de corrupción en Colombia, lo que indica que existen mecanismos y procedimientos legales para llevar a cabo las investigaciones, pero estos no son suficientes para acabar con este flagelo que tanto perjudica la función pública y el conglomerado social de la perla del norte [17].

Frente a las diferentes acciones y estrategias implementadas por el gobierno municipal en los últimos dos períodos, en materia de promoción y participación ciudadana, junto a los procesos de veeduría y control social a lo público, avocados por la Alcaldía de Cúcuta y Órganos de Control del Estado, se logró verificar la existencia de algunos procesos de veeduría ciudadana aplicados a la veeduría al PAE, veeduría la doble calzada Cúcuta Pamplona, veeduría los megaproyectos y/o megaobras que se vienen desarrollando en el municipio Cúcuta como lo son la Obra de Cuatro Vientos, Parque Cristo Rey y Redoma del Indio entre las más destacadas. Igualmente se logró visualizar que de la contratación realizada por el Municipio de Cúcuta, durante los años 2013 al 2018, tan solo el $1.5 \%$, fueron objeto de control, seguimiento y monitoreo a través de procesos de veeduría ciudadana debidamente formalizados y/o registradas, situación que indica que la participación ciudadana en los proceso de contratación no es representativa, por lo que es una obligación actuar y ejercer el control social a lo público como lo ordena la Ley 1757 de 2015, por parte de todos los ciudadanos. [18]. 
Finalmente, esta investigación propone mecanismos y/o alternativas para mejorar el indicador de participación ciudadana y control social a lo público, dejando una propuesta visionaria y de prospectiva para mejorar el indicador de participación ciudadana, que inicialmente está encaminada hacia los adolescentes y jóvenes de educación media, específicamente aplicada a la Institución Educativa San Francisco de Sales, para estudiantes el nivel medio correspondiente a los grados 8 al 11, quienes podrán contar con herramientas e instrumentos importantes para enriquecer sus conocimientos en materia de Participación Ciudadana y Control Social a lo Público, ayudas que al requerirse, las obtendrán de manera inmediata ya que se encuentran "a la mano" y a través de medios electrónicos, con la entrega y aplicación del Manual del Contralor Estudiantil, soporte documental que les brindará conocimientos básicos y los empoderará en las acciones referentes a la participación ciudadana.

Recomendaciones para mejorar la calidad de vida

Como toda innovación social educativa, este trabajo busca encontrar oportunidades que permitan la mejora continua, el mejoramiento de la calidad de vida de los educandos, enriquecer la cultura ciudadana, sobre la base de un ejercicio del control social a lo público, que redundará en beneficios, oportunidades y mejoramiento del tejido social de las regiones, que hoy se encuentran inmersas en las necesidades básicas insatisfechas que requieren el apalancamiento de la participación ciudadana para que ese gestor público realice los procesos y procedimientos al estructurar sus planes estratégicos y gestión como orden a la le y así lograr la obtención del beneficio social con resultados e indicadores que demuestren eficiencia, eficacia, y efectividad.
Esta investigación que fortalecerá el aprendizaje en la institución indagada y a las que se pueda extender este estudio, es por ello, que se sugiere que las instituciones públicas competentes como lo es la Secretaria de Educación Municipal en coordinación con la Contraloría Municipal de Cúcuta, empoderen su talento humano y le concedan todos los elementos y herramientas requeridas conforme al espacio educativo donde se desenvuelven, para que el docente pueda contar con una metodología propia para la enseñanza de una cultura ciudadana sobre el control social a lo público, y así el alumno cree actitudes para ser más autónomo y responsable de su propio aprendizaje.

Otro aspecto fundamental e importante para poner en marcha en todas las I.E. son las "Charlas, foros, mesas de trabajo, y otras que se requieran", entre estudiantes directivos docentes, órganos de control y Secretaria de Educación sobre mecanismos de participación ciudadana y control social a lo público, como un área electiva, en el respetico PEI, (Plan Educativo Anual e Institucional), lo cual nos permitirá obtener y realizar intercambios de conocimiento, y necesidades de tipo social de los jóvenes.

"La participación ciudadana consiste, en último término, en la instauración de un proceso de trabajo compartido. Un proceso de toma de conciencia en el que diferentes actores sociales de un determinado territorio hacen frente a su realidad, determinan sus pautas de reivindicación y de mejora, encargándose de llevar a cabo cuantos proyectos concreten el proceso en marcha. Cuando el proceso es educativo el proyecto o los proyectos particulares se ponen al servicio del proceso global y es el mismo proceso el que va apuntalando y evaluando cada proyecto y su significado [7]. 
El control social a lo publico educa, mejora nuestra calidad de vida, y es responsabilidad de todos.

\section{2}

\section{Referencias}

[1] N. Cosoy, "Colombia: el gobierno y las FARC firman un nuevo acuerdo de paz" BBC Mundo, 2016. [En línea]. Disponible em: https://www.bbc.com/mundo/noticias-americalatina-37965381

[2] P. Martínez, “Corrupciónvisible"RevistaDigital Semana, 2017. Recuperado de: https://www. semana.com/opinion/articulo/corrupcion-enel-sector-publico-en-colombia/523250

[3] Alcaldía de Bogotá, "Normas orientadas a fortalecer los mecanismos de prevención, investigación y sanción de actos de corrupción y la efectividad del control de la gestión pública"m 2011. [En línea]. Disponible en: https://www. alcaldiabogota.gov.co/sisjur/normas/Normal. jsp?i=43292

[4] A. Aguilar Barreto, C. Soto Rojas, y J.M Castaño Quijano, "Políticas públicas de juventud: factor de desarrollo y participación en Norte de Santander y San José de Cúcuta, Mundo Fesc, vol. 5, n. ${ }^{\circ}$ 10, pp. 69-74, feb. 2016.

[5] E. Jiménez y A. Martínez. "Contraloría social y decisiones de política pública en el ámbito local: binomio necesario", 2016. [En línea]. Disponible en: https://www.uv.mx/iiesca/ files/2017/03/24CA201602.pdf

[6] M. Hernández. "Participación ciudadana y el rescate de la ciudad", Revista INVI, vol. 22, no. 59, 2007

[7] L.A Aranguren, "La participación ciudadana, posibilidades y retos", Aposta: Revista de ciencias sociales, $\mathrm{n}^{\circ} .22,2005$

[8] J.A Hurtado Mosquera, L. Hinestroza Cuesta, "La participación democrática en Colombia un derecho en evolución 1", Justicia Juris, vol. 12, no. 2, pp. 59-76, 2016
[9] C.F Echeverri Jiménez, "La participacion ciudadana en Colombia reflexiones desde la perspectiva constitucional y la normatividad estatutaria". Universidad de Antioquia, 2010

[10] A.D. Arana -Gutiérrez, L.A. Duarte y O.M. Giraldo-Alzate, "Visión crítica al derecho positivo: la seguridad social bajo el influjo modelo neoliberal", Revista Criterio Libre Juridico, vol. 15, no. 1, 2018

[11] Conexión Capital, "La abstención electoral en Colombia", 2018. [En línea]. Disponible en: https://conexioncapital.co/la-abstencionelectoral-en-colombia/

[12] F.G Ssolano Villamizar, R.A. Rincón Méndez, D.E. Herrera Ziñiga y A.C. Sabogal Suescun, "Formulación de un proyecto social enfocado a promover la participación ciudadana, Universidad Católica de Colombia, 2018

[13] Colección Cuadernos de Transparencia No 19, "Segunda encuesta nacional sobre prácticas contra el soborno en empresas colombianas" Colombia- América del Sur, Noviembre de 2010

[14] Departamento Administrativo de la Función Pública, "Integración De Planes Institucionales, Decreto 612”, 2018. [En línea]. Disponible en: https://dapre.presidencia.gov.co/normativa/ normativa/DECRETO\%20612\%20DEL\%20 04\%20DE\%20ABRIL\%20DE\%202018.pdf

[15] A. Caballero Tovío, "Grado de articulación de las estrategias consignadas en el Plan Nacional de Desarrollo 2014-2018 con la situación del sector industrial colombiano", Enfoque Disciplinario, vol. 1, pp. 14-20, 2016.

[16] Congreso República de Colombia, "Ley de Transparencia y del Derecho de Acceso a la Información Pública Nacional", 2014. [En línea], Disponible en: http://www.secretariasenado. gov.co/senado/basedoc/ley_1712_2014.html 
[17] A.R. Vargas Malaver, "Sistema de comtrol de control fiscal colombiano en la búsqueda de la construcción de una administración pública eficiente CGR 1990-2010", Universidad Libre de Colombia, 2012

[18] Congreso de la República, "Ley estatutaria 1757 de 2015”, 2015. [En línea]. Disponible en: https://www.funcionpublica.gov.co/eva/ gestornormativo/norma.php? $\mathrm{i}=65335$ 